\title{
MÖBIUS MAPS BETWEEN ULTRAMETRIC SPACES ARE LOCAL SIMILARITIES
}

\author{
Zair Ibragimov \\ California State University, Department of Mathematics \\ Fullerton, CA 92831, U.S.A.; zibragimov@fullerton.edu
}

\begin{abstract}
We show that Möbius maps between ultrametric spaces are local similarities. The proof is based on the notions of chordal ultrametric and normalization, which we introduce in this paper.
\end{abstract}

\section{Introduction}

A metric space $(X, d)$ is called an ultrametric space if $d$ satisfies the strong triangle inequality: $d(x, y) \leq \max \{d(x, z), d(y, z)\}$ for all $x, y, z \in X$. Any compact perfect ultrametric space is homeomorphic to the ternary Cantor set $C$; it is quasisymmetric to $C$ if and only if it is complete, doubling and uniformly perfect (see [4, Proposition 15.11]). It is a well-known fact that balls in ultrametric spaces possess some special properties not shared by balls in general metric spaces. For example, (1) balls are both open and closed; (2) any two balls are either disjoint or one is contained in the other; (3) every point in a ball can be its center; (4) every ball is a union of disjoint balls; (5) the diameter of a ball is less than or equal to its radius. These properties are easily derived from the strong triangle property (see, for example, $[4,14,15])$. Well-known examples of ultrametric spaces are the fields of $p$-adic numbers $\mathbf{Q}_{p}$, which are the object of study in $p$-adic Analysis and Fractal Geometry (see $[10,11,12,13,16]$ and the references therein). A brief review of some selected topics in $p$-adic mathematical physics can be found in ([5]).

Ultrametric spaces also arise as the boundaries at infinity of metric trees and more general Gromov 0-hyperbolic spaces (see, for instance, [2, 3, 8, 18]). Recently, Hughes has studied connections between metric trees and ultrametric spaces from a categorical point of view. He established an equivalence from the category of geodesically complete, rooted metric trees and the equivalence classes of isometries at infinity, to the category of complete ultrametric spaces of finite diameter and the local similarity equivalences ([8, Main Theorem]).

The work of Hughes shows that local similarities are a natural class of maps to study on ultrametric spaces. It is also known that isometries at infinity between Gromov 0-hyperbolic spaces induce Möbius maps between their boundaries at infinity. A natural question is: what is the connection between local isometries and Möbius maps on ultrametric spaces? Our main result in this paper provides an answer to this question. Namely, we show that every Möbius map between any two ultrametric spaces is a local similarity.

doi:10.5186/aasfm.2012.3725

2010 Mathematics Subject Classification: Primary 30C65; Secondary 05C25.

Key words: Ultrametric space, chordal ultrametric, local similarity, Möbius map. 
In [8] Hughes posed the problem of classifying complete ultrametric spaces up to local similarities. Since Möbius maps on ultrametric spaces are local similarities (Theorem 5.2), classifying ultrametric spaces up to Möbius maps would be an important step in the direction of Hughes' Problem. Section 2 contains basic concepts and some preliminary results on ultrametric spaces. In Sections 3 we introduce the concepts of chordal ultrametrics and inversions on ultrametric spaces. In Section 4 we introduce the concept of normalization for ultrametric spaces. These concepts are used in the proof of our main result in Section 5.

\section{Ultrametric spaces}

By an ultrametric on a set $X$ we mean a metric $d$ on $X$ satisfying the strong triangle inequality

$$
d(x, y) \leq d(x, z) \vee d(z, y)
$$

for $x, y, z \in X$. Here and, in what follows, we set $r \vee s=\max \{r, s\}$. Clearly, an ultrametric is a metric and if $d$ is an ultrametric, then so is $d^{s}$ for all $s \in(0, \infty)$, where $d^{s}(x, y)=(d(x, y))^{s}$. In fact, a metric $d$ is an ultrametric if and only if $d^{s}$ is a metric for every $s>0$ (see, for example, [7, Corollary 3.4]).

The pair $(X, d)$, where $d$ is an ultrametric, is called an ultrametric space. An open ball centered at $x \in X$ and of radius $r>0$ is denoted by $B(x, r)$ and the closed ball is denoted by $\bar{B}(x, r)$. The crossratio of the quadruple $x, y, z, w \in X$ of ordered points with $x \neq z$ and $y \neq w$ is defined by

$$
[x, y, z, w]=\frac{d(x, y) d(z, w)}{d(x, z) d(y, w)} .
$$

Let $(X, d)$ and $\left(X^{\prime}, d^{\prime}\right)$ be ultrametric spaces and let $f: X \rightarrow X^{\prime}$ be a homeomorphism. The map $f$ is called a similarity if there exists $\lambda>0$ such that $d^{\prime}(f(x), f(y))=\lambda d(x, y)$ for all $x, y \in X$. We also say that $f$ is a $\lambda$-similarity. If $\lambda=1$, we say that $f$ is an isometry. The map $f$ is called a local similarity if for each $x \in X$ there exist $\epsilon>0$ and $\lambda>0$ such that the restriction $f \mid: B(x, \epsilon) \rightarrow B(f(x), \lambda \epsilon)$ is a surjective $\lambda$-similarity. Finally, we say that $f$ is Möbius if $[f(x), f(y), f(z), f(w)]=[x, y, z, w]$ for all $x, y, z, w \in X$.

Suppose that $(X, d)$ is an ultrametric space. Note that given $x, y, z \in X$, the strong triangle inequality implies that the two larger of the distances $d(x, y), d(x, z)$ and $d(y, z)$ are equal. This property is often expressed by saying that every triangle in $X$ is isosceles and, moreover, the length of the base is less than or equal to the length of the equal sides. The strong triangle inequality implies the following conditions on quadruples. Recall that a distance function on a set $X$ is a nonnegative symmetric function on the product set $X \times X$.

Lemma 2.1. If a distance function $d$ on $X$ satisfies the strong triangle inequality, then for each quadruple $x, y, z, w \in X$ the two larger of the sums

$$
d(x, y)+d(z, w), \quad d(x, z)+d(y, w), \quad d(x, w)+d(y, z)
$$

and the two larger of the products

$$
d(x, y) d(z, w), \quad d(x, z) d(y, w), \quad d(x, w) d(y, z)
$$

are equal. Moreover, $d(x, y)+d(z, w)=d(x, z)+d(y, w) \geq d(x, w)+d(y, z)$ if and only if $d(x, y) d(z, w)=d(x, z) d(y, w) \geq d(x, w) d(y, z)$. 
Proof. Without loss of generality we can assume that $d(x, y) \geq d(x, z) \vee d(y, z) \vee$ $d(x, w) \vee d(y, w) \vee d(z, w)$. Then $d(x, y)=d(x, z) \vee d(y, z)$ and $d(x, y)=d(x, w) \vee$ $d(y, w)$. There are four possibilities.

Case 1: $d(x, z) \geq d(y, z)$ and $d(x, w) \geq d(y, w)$. Then $d(x, y)=d(x, z)=d(x, w)$.

Case 2: $d(x, z) \leq d(y, z)$ and $d(x, w) \leq d(y, w)$. Then $d(x, y)=d(y, z)=d(y, w)$.

Case 3: $d(x, z) \geq d(y, z)$ and $d(x, w) \leq d(y, w)$. Then $d(z, w) \leq d(y, z) \vee$ $d(y, w) \leq d(x, z) \vee d(y, w)=d(x, y)$ and hence $d(x, y)=d(x, z)=d(y, w)=d(z, w)$.

Case 4: $d(x, z) \leq d(y, z)$ and $d(x, w) \geq d(y, w)$. Then $d(z, w) \leq d(x, z) \vee$ $d(x, w) \leq d(y, z) \vee d(x, w)=d(x, y)$ and hence $d(x, y)=d(y, z)=d(x, w)=d(z, w)$. In all cases we obtain (2.2) and (2.3) as well as the second part of the lemma.

The first part of Lemma 2.1 is equivalent to the following inequalities

$$
d(x, y)+d(z, w) \leq[d(x, z)+d(y, w)] \vee[d(x, w)+d(y, z)]
$$

and

$$
d(x, y) d(z, w) \leq[d(x, z) d(y, w)] \vee[d(x, w) d(y, z)]
$$

respectively. Observe that the conditions (2.4) and (2.5) imply that ultrametric spaces are both Gromov 0-hyperbolic and ptolemaic (see $[3,9]$ ).

If, in Lemma 2.1, $d$ is an ultrametric on $X$, then the lemma also follows from Theorem $3.2[6]$ when the latter is applied to the four-point ultrametric space $(\{x, y, z, w\}$, $d)$. In this paper we apply this lemma only for ultrametric spaces. In our upcoming paper "hyperbolic fillings for ultrametric spaces" we apply this lemma for more general distance functions that satisfy the strong triangle inequality, justifying its present formulation.

\section{Chordal ultrametrics and inversions}

Suppose that $(X, d)$ is any ultrametric space. If $X$ is unbounded, we let $\hat{X}=$ $X \cup\{\infty\}$ be its one-point extension. For simplicity we put $\hat{X}=X$ if $X$ is bounded. The crossratio is extended to $\hat{X}$ by putting $d(x, \infty) / d(y, \infty)=1$ for all $x, y \in X$.

Let $V$ be an arbitrary subset of $X$ with $0<\operatorname{diam}(V)<\infty$. For $x \in X$ we put

$$
m(x, V)=\sup _{v \in V} d(x, v) .
$$

If $X$ is an unbounded ultrametric space, then for each fixed $x \in X$ we have $d(x, y) /$ $m(y, V)=1$ for all $y \in X \backslash \bar{B}(x, m(x, V))$. Indeed, for each $v \in V$ we have $d(y, v) \leq$ $d(y, x) \vee d(x, v) \leq d(y, x) \vee m(x, V)=d(x, y)$. Taking the supremum over all $v \in V$ we obtain that $m(y, V) \leq d(x, y)$. On the other hand, for each $v \in V$ we have $d(x, v) \leq d(y, v)$ for otherwise using the strong triangle inequality we would have $d(x, y)=d(x, v) \leq m(x, V)$ contradicting to the fact that $y \in X \backslash \bar{B}(x, m(x, V))$. Hence $d(x, y) \leq d(x, v) \vee d(y, v)=d(y, v) \leq m(y, V)$. Thus, $d(x, y)=m(y, V)$ proving the claim. By continuity, we set $d(x, \infty) / m(\infty, V)=1$.

Lemma 3.1. Let $(X, d)$ be an ultrametric space and let $V$ be any subset of $X$ with $0<\operatorname{diam}(V)<\infty$. Then $m(x, V) \geq \operatorname{diam}(V)$ for all $x \in X$. The equality holds if $x \in V$. Moreover, if $m(x, V)>\operatorname{diam}(V)$, then $m(x, V)=d(x, v)$ for all $v \in V$.

Proof. Given $v_{1}, v_{2} \in V$ and $x \in X$, we have $d\left(v_{1}, v_{2}\right) \leq d\left(x, v_{1}\right) \vee d\left(x, v_{2}\right) \leq$ $m(x, V)$. Taking the supremum over all $v_{1}, v_{2} \in V$, we obtain $m(x, V) \geq \operatorname{diam}(V)$. 
Clearly, if $x \in V$ then $m(x, V) \leq \operatorname{diam}(V)$ and hence $m(x, V)=\operatorname{diam}(V)$. If $m(x, V)>\operatorname{diam}(V)$, then there exists $y \in V$ such that $d(x, y)>\operatorname{diam}(V)$. Given arbitrary $v \in V$, since the two larger of the distances $d(x, y), d(y, v)$ and $d(x, v)$ are equal and since $d(y, v) \leq \operatorname{diam}(V)<d(x, y)$, we have $d(x, y)=d(x, v)$. Since $v$ is arbitrary, we obtain $d\left(x, v_{1}\right)=d\left(x, v_{2}\right)$ for all $v_{1}, v_{2} \in V$ and hence $m(x, V)=d(x, v)$ for all $v \in V$.

Now we define a distance function $d_{V}$ on $\hat{X}$ by

$$
d_{V}(x, y)= \begin{cases}\frac{\operatorname{diam}(V) d(x, y)}{m(x, V) m(y, V)} & \text { if } x, y \in X \\ \frac{\operatorname{diam}(V)}{m(x, V)} & \text { if } x \in X \text { and } y=\infty \\ 0 & \text { if } x=y=\infty\end{cases}
$$

Observe that if $X$ is bounded, then for $V=X$ we have $d_{V}(x, y)=d(x, y) / \operatorname{diam}(X)$ for all $x, y \in X$. For unbounded $X$, it can be shown that the space $\left(\hat{X}, d_{V}\right)$ is homeomorphic to the one-point compactification of $X$ if and only if $X$ is proper (that is, every closed bounded subset of $X$ is compact).

Theorem 3.3. Let $(X, d)$ be an ultrametric space and let $V \subset X$ with $0<$ $\operatorname{diam}(V)<\infty$. Then

(1) the distance function $d_{V}$ is an ultrametric on $\hat{X}$;

(2) for all $x, y \in \hat{X}$ we have $d_{V}(x, y) \leq 1$. Equality holds for $x, y \in V$ with $d(x, y)=\operatorname{diam}(V)$ as well as for $x \in X, y \in V$ with $m(x, V)>\operatorname{diam}(V)$;

(3) the identity map id: $(X, d) \rightarrow\left(X, d_{V}\right)$ is both Möbius and a local similarity;

(4) if $(X, d)$ is complete, then so is $\left(\hat{X}, d_{V}\right)$.

Proof. To prove (1), we observe that $d_{V}(x, y)=0$ if and only if $x=y$. We need to show that $d_{V}$ satisfies the strong triangle inequality. By continuity it is enough to consider only the points $x, y, z \in X$. Without loss of generality we can assume that $d_{V}(x, y) \geq d_{V}(x, z) \vee d_{V}(y, z)$. Then

$$
d(x, y) m(z, V) \geq d(x, z) m(y, V) \vee d(y, z) m(x, V) .
$$

For any $v \in V$ using Lemma 2.1 we obtain

$$
d(x, y) d(z, v) \leq d(x, z) d(y, v) \vee d(y, z) d(x, v) \leq d(x, z) m(y, V) \vee d(y, z) m(x, V) .
$$

Taking the supremum over all $v \in V$ we obtain

$$
d(x, y) m(z, V) \leq d(x, z) m(y, V) \vee d(y, z) m(x, V),
$$

which is equivalent to

$$
d_{V}(x, y) \leq d_{V}(x, z) \vee d_{V}(y, z)
$$

Thus, the two larger of the distances $d_{V}(x, y), d_{V}(x, z), d_{V}(y, z)$ are equal, establishing the strong triangle property.

To prove (2), for all $v_{1}, v_{2} \in V$ and $x, y \in X$, using Lemma 2.1 we obtain

$$
d(x, y) d\left(v_{1}, v_{2}\right) \leq d\left(x, v_{1}\right) d\left(y, v_{2}\right) \vee d\left(x, v_{2}\right) d\left(y, v_{1}\right) \leq m(x, V) m(y, V)
$$

and hence $d_{V}(x, y) \leq 1$. If $x \in X$ and $y=\infty$, then using Lemma 3.1 we obtain $d_{V}(x, y)=\operatorname{diam}(V) / m(x, V) \leq 1$. Finally, for $x, y \in V$ with $d(x, y)=\operatorname{diam}(V)$ and for $x \in X, y \in V$ with $m(x, V)>\operatorname{diam}(V)$, Lemma 3.1 implies that $d_{V}(x, y)=1$. 
To prove (3), put $\epsilon=\operatorname{diam}(V)$. For each $x \in X$ we let

$$
\lambda_{1}=\frac{\operatorname{diam}(V)}{[\operatorname{diam}(B(x, \epsilon) \cup V)]^{2}} .
$$

Then for $y \in B(x, \epsilon)$ we have $m(x, V)=m(y, V)$. Indeed, using Lemma 3.1 we obtain

$$
m(y, V)=\sup _{v \in V} d(y, v) \leq \sup _{v \in V}[d(x, y) \vee d(x, v)] \leq \operatorname{diam}(V) \vee m(x, V)=m(x, V) .
$$

Similarly, $m(x, V) \leq m(y, V)$. The common value of $m(y, V)$ for $y \in B(x, \epsilon)$ is equal to $\operatorname{diam}(B(x, \epsilon) \cup V)$. Hence

$$
d_{V}(y, z)=\frac{\operatorname{diam}(V) d(y, z)}{m(y, V) m(z, V)}=\lambda_{1} d(y, z) \quad \text { for all } y, z \in B(x, \epsilon) .
$$

Thus, $\operatorname{id}_{V}$ is both a homeomorphism and a local similarity. Finally, it is an immediate consequence of the definitions that the identity map is Möbius.

To prove (4), let $\left\{x_{i}\right\}$ be a Cauchy sequence in $\left(\hat{X}, d_{V}\right)$. That is, $d_{V}\left(x_{i}, x_{j}\right)$ tends to 0 as $i \rightarrow \infty$ and $j \rightarrow \infty$. Without loss of generality we can assume that $\infty \notin\left\{x_{i}\right\}$. If the set $\left\{m\left(x_{i}, V\right): i=1,2, \ldots\right\}$ is bounded, then it is easy to see that $d\left(x_{i}, x_{j}\right)$ tends to 0 as $i \rightarrow \infty$ and $j \rightarrow \infty$. Then $\left\{x_{i}\right\}$ is a Cauchy sequence in $(X, d)$ and hence converges to a point in $X \subset \hat{X}$. If the set $\left\{m\left(x_{i}, V\right): i=1,2, \ldots\right\}$ is unbounded, then $d_{V}\left(x_{i}, \infty\right)=\operatorname{diam}(V) / m\left(x_{i}, V\right)$ and hence $\infty$ is a cluster point of $\left\{x_{i}\right\}$. Since $\left\{x_{i}\right\}$ is a Cauchy sequence, $\left\{x_{i}\right\}$ converges to $\infty$, as required.

We refer to the distance functions $d_{V}$ as the chordal ultrametrics. As the identity map in Theorem 3.3 is a local similarity it maps balls of small enough radius to balls. The next lemma says that it does not map every ball to a ball.

Lemma 3.4. let $(X, d)$ be an ultrametric space and $V \subset X$ with $0<\operatorname{diam}(V)<$ $\infty$. If $B$ is a ball in $(X, d)$ not containing $V$, then $B$ is a ball in $\left(X, d_{V}\right)$. If $V \subset B$, then $B$ is not a ball in $\left(X, d_{V}\right)$ except for $B=X$.

Proof. Suppose that $B$ is a ball in $(X, d)$. If $V \backslash B \neq \emptyset$, then $m(x, V)=m(y, V)=$ $\operatorname{diam}(V \cup B)$ for all $x, y \in B$ and hence $d_{V}(x, y)=\operatorname{diam}(V) d(x, y) / \operatorname{diam}(V \cup B)$. Therefore $B$ is a ball of radius $\operatorname{diam}(V) \operatorname{diam}(B) / \operatorname{diam}(V \cup B)$ in $\left(X, d_{V}\right)$. Now let $V \subset B$. Let $v \in V$ and $x \in B$ be such that $d(v, x)=m(x, V)$. Then $d_{V}(x, v)=1$, that is, $\operatorname{diam}(B)=1$ in $\left(X, d_{V}\right)$. Since $\operatorname{diam}(X)=1$ with respect to $d_{V}$, we conclude that $B$ is not a ball in $\left(X, d_{V}\right)$ except when $B=X$.

Next, we introduce the concept of inversions on ultrametric spaces. For each $p \in X$ and $r>0$, we define a distance function $d_{p, r}$ on $X_{p}=\hat{X} \backslash\{p\}$ by

$$
d_{p, r}(x, y)=\frac{r^{2} d(x, y)}{d(x, p) d(y, p)} .
$$

The $\operatorname{maps}_{\mathrm{id}}$ will be referred to as the inversions about $p$. Note that if $p$ is not an isolated point, then the space $\left(X_{p}, d_{p, r}\right)$ is unbounded.

Lemma 3.6. Suppose that $(X, d)$ is an ultrametric space and let $p \in X$ and $r>0$. Then the distance function $d_{p, r}$ is an ultrametric. Moreover, the identity map $\operatorname{id}_{p}:\left(X_{p}, d\right) \rightarrow\left(X_{p}, d_{p, r}\right)$ is both Möbius and a local similarity. 
Proof. By Lemma 2.1 we have

$$
d(x, y) d(z, p) \leq d(x, z) d(y, p) \vee d(y, z) d(x, p)
$$

for all $x, y, z \in X$. Dividing all the terms by $d(x, p) d(y, p) d(z, p)$ we obtain

$$
\frac{d(x, y)}{d(x, p) d(y, p)} \leq \frac{d(x, z)}{d(x, p) d(z, p)} \vee \frac{d(y, z)}{d(y, p) d(z, p)}
$$

Hence the distance function $d_{p, r}$ is an ultrametric.

To prove the second part, let $\epsilon_{2} \in(0, d(x, p)]$ and $\lambda_{2}=\left[r / d(x, p]^{2}\right.$ for $x \in X_{p}$. Then for all $y \in B\left(x, \epsilon_{2}\right)$ we have $d(y, p)=d(x, p)$ and hence

$$
d_{p, r}(y, z)=\frac{r^{2} d(y, z)}{d(y, p) d(z, p)}=\lambda_{2} d(y, z) \quad \text { for all } \quad y, z \in B\left(x, \epsilon_{2}\right) .
$$

Hence $\mathrm{id}_{p}$ is both a homeomorphism and a local similarity. Finally, it follows from the definitions that $\mathrm{id}_{p}$ is also Möbius.

\section{Normalization}

Let $X=(X, d)$ be any ultrametric space and let $p=\left\{p_{1}, p_{2}, p_{3}\right\}$ be a triple of distinct points in $X$ with $d\left(p_{1}, p_{2}\right) \leq d\left(p_{3}, p_{1}\right)=d\left(p_{3}, p_{2}\right)$. Define

$$
\chi_{p}(x, y)=\frac{d\left(p_{1}, p_{2}\right) d(x, y)}{\left[d\left(x, p_{1}\right) \vee d\left(x, p_{2}\right)\right]\left[d\left(y, p_{1}\right) \vee d\left(y, p_{2}\right)\right]} .
$$

Observe that $\chi_{p}=d_{V}$, where $V=\left\{p_{1}, p_{2}\right\}$ and $d_{V}$ is as in (3.2). Hence by Theorem 3.3 the space $\left(X, \chi_{p}\right)$ is an ultrametric space of diameter equal to 1 , and that the identity map $\mathrm{I}_{p}:(X, d) \rightarrow\left(X, \chi_{p}\right)$ is both Möbius and a local similarity. A simple computation shows that

$$
\chi_{p}\left(p_{1}, p_{2}\right)=\chi_{p}\left(p_{1}, p_{3}\right)=\chi_{p}\left(p_{2}, p_{3}\right)=1 .
$$

We refer to the space $X_{p}=\left(X, \chi_{p}\right)$ as the normalization of $X$ with respect to the triple $p=\left\{p_{1}, p_{2}, p_{3}\right\}$. Notice that the normalization of $X_{p}$ with respect to the triple $p=\left\{p_{1}, p_{2}, p_{3}\right\}$ is the space $X_{p}$ itself.

We discuss some properties of $X_{p}$. Put $r=d\left(p_{1}, p_{2}\right)$. Balls in $X_{p}$ are denoted using a subscript $p$. Given $x_{0} \in X$, for all $x, y \in B\left(x_{0}, r\right)$ we have (see the proof of Theorem 3.3)

$$
\chi_{p}(x, y)=\frac{r}{\left[d\left(x_{0}, p_{1}\right) \vee d\left(x_{0}, p_{2}\right)\right]^{2}} d(x, y) .
$$

Since $d\left(x, p_{1}\right) \vee d\left(x, p_{2}\right)=r$ for each $x \in \bar{B}\left(p_{i}, r\right)(i=1,2)$, it follows that the restriction of the identity map $\mathrm{I}_{p}$ to $\bar{B}\left(p_{1}, r\right)$ is a $(1 / r)$-similarity, which maps $\bar{B}\left(p_{1}, r\right)$ in $X$ onto $\bar{B}_{p}\left(p_{1}, 1\right)$ in $X_{p}$.

Next, the closed ball $\bar{B}\left(p_{1}, r\right)$ decomposes into the union of disjoint balls of radius $r$. More precisely, let $\left\{x_{i}: i \in I\right\}$ be the set of all points in $\bar{B}\left(p_{1}, r\right)$ with $d\left(x_{i}, x_{j}\right)=r$ for $i \neq j$, where $I$ is some indexing set. Note that the set $I$ is countable if $X$ is separable and is finite if $\bar{B}\left(p_{1}, r\right)$ is compact. Then

$$
\bar{B}\left(p_{1}, r\right)=\bigcup_{i \in I} B\left(x_{i}, r\right)
$$

and hence

$$
\bar{B}_{p}\left(p_{1}, 1\right)=\bigcup_{i \in I} B_{p}\left(x_{i}, 1\right) .
$$


Note that $\mathrm{I}_{p}\left(B\left(x_{i}, r\right)\right)=B_{p}\left(x_{i}, 1\right)$.

The complement of $\bar{B}\left(p_{1}, r\right)$, that is, the set $X \backslash \bar{B}\left(p_{1}, r\right)$ (assuming it is not empty), is mapped by $\mathrm{I}_{p}$ onto a unit ball $B_{p}(x, 1)$, where $x \in X \backslash \bar{B}\left(p_{1}, r\right)$ is any point. Indeed, we have $d\left(x, p_{1}\right)=d\left(x, p_{2}\right)>r$ and if $y \in X \backslash \bar{B}\left(p_{1}, r\right)$, then $d\left(y, p_{1}\right)=$ $d\left(y, p_{2}\right)>r$. Hence

$$
\chi_{p}(x, y)=\frac{r d(x, y)}{d\left(x, p_{1}\right) d\left(y, p_{1}\right)}<\frac{\min \left\{d\left(x, p_{1}\right), d\left(y, p_{1}\right)\right\}\left[d\left(x, p_{1}\right) \vee d\left(y, p_{1}\right)\right]}{d\left(x, p_{1}\right) d\left(y, p_{1}\right)}=1
$$

so that $y \in B_{p}(x, 1)$. Conversely, if $y \in \bar{B}\left(p_{1}, r\right)$, then $d(x, y)=d\left(x, p_{1}\right)>d\left(y, p_{1}\right)$. Hence

$$
\chi_{p}(x, y)=\frac{r d(x, y)}{d\left(x, p_{1}\right)\left[d\left(y, p_{1}\right) \vee d\left(y, p_{2}\right)\right]}=\frac{r}{d\left(y, p_{1}\right) \vee d\left(y, p_{2}\right)} \geq 1
$$

so that $y \in X_{p} \backslash B_{p}(x, 1)$. Moreover, the map $\mathrm{I}_{p}$ acts as a local similarity on $X \backslash$ $\bar{B}\left(p_{1}, r\right)$. Indeed, given $x_{0} \in X \backslash \bar{B}\left(p_{1}, r\right)$, since $\left.d\left(x_{0}\right), p_{1}\right)=d\left(x_{0}, p_{2}\right)>r$, using the strong triangle inequality we obtain $d\left(x, p_{1}\right) \vee d\left(x, p_{2}\right)=d\left(x_{0}, p_{1}\right)$ for each $x \in$ $B\left(x_{0}, r\right)$. Hence for each $x, y \in B\left(x_{0}, r\right)$ we have

$$
\chi_{p}(x, y)=\lambda\left(x_{0}\right) d(x, y), \quad \text { where } \lambda\left(x_{0}\right)=\frac{r}{\left[d\left(x_{0}, p_{1}\right)\right]^{2}} .
$$

We say that an ultrametric space $(X, d)$ is normalized if there exist $p_{1}, p_{2}, p_{3} \in$ $X$ such that $d\left(p_{1}, p_{2}\right)=d\left(p_{1}, p_{3}\right)=d\left(p_{2}, p_{3}\right)=\operatorname{diam}(X)=1$. We conclude that in classifying ultrametric spaces, up to a Möbius map, one can consider only the normalized ultrametric spaces. Analogously, in classifying ultrametric spaces, up to a local similarity, one can consider only the normalized ultrametric spaces.

\section{Möbius maps between ultrametric spaces}

Our goal in this section is to prove our main result that Möbius maps between ultrametric spaces are local similarities. We say that a triple $\{x, y, z\}$ of points in an ultrametric space $(X, d)$ is diametrical if $d(x, y)=d(x, z)=d(y, z)=\operatorname{diam}(X)$. We begin with the following preliminary lemma.

Lemma 5.1. Let $(X, d)$ and $\left(X^{\prime}, d^{\prime}\right)$ be ultrametric spaces of diameters equal to 1. If $f$ is a Möbius map between $X$ and $X^{\prime}$ that maps a diametrical triple in $X$ to a diametrical triple in $X^{\prime}$, then $f$ is an isometry.

Proof. Let $\left\{x_{1}, x_{2}, x_{3}\right\}$ be a diametrical triple in $X$ such that $\left\{f\left(x_{1}\right), f\left(x_{2}\right), f\left(x_{3}\right)\right\}$ is a diametrical triple in $X^{\prime}$. That is, $d\left(x_{i}, x_{j}\right)=1=d^{\prime}\left(f\left(x_{i}\right), f\left(x_{j}\right)\right)$ for $i \neq j$. We will show that

$$
d^{\prime}(f(x), f(y))=d(x, y) \quad \text { for all } x, y \in X .
$$

We begin by showing that $d^{\prime}\left(f(x), f\left(x_{i}\right)\right)=d\left(x, x_{i}\right)$ for any $x \in X$ and $i \in\{1,2,3\}$. Let $x \in X$ be given. If $d\left(x, x_{i}\right)=1$ for all $i \in\{1,2,3\}$, then for $j, k \in\{1,2,3\}$ with $i \neq j \neq k \neq i$ we have

$$
\frac{d^{\prime}\left(f(x), f\left(x_{i}\right)\right)}{d^{\prime}\left(f(x), f\left(x_{j}\right)\right)}=\frac{d^{\prime}\left(f(x), f\left(x_{i}\right)\right) d^{\prime}\left(f\left(x_{j}\right), f\left(x_{k}\right)\right)}{d^{\prime}\left(f(x), f\left(x_{j}\right)\right) d^{\prime}\left(f\left(x_{i}\right), f\left(x_{k}\right)\right)}=\frac{d\left(x, x_{i}\right) d\left(x_{j}, x_{k}\right)}{d\left(x, x_{j}\right) d\left(x_{i}, x_{k}\right)}=1 .
$$

Hence $d^{\prime}\left(f(x), f\left(x_{i}\right)\right)=d^{\prime}\left(f(x), f\left(x_{j}\right)\right)$. Since $d^{\prime}\left(f\left(x_{i}\right), f\left(x_{j}\right)\right)=1$, from the strong triangle inequality we obtain $d^{\prime}\left(f(x), f\left(x_{i}\right)\right)=1$ for any $i \in\{1,2,3\}$, as required. If 
$d\left(x, x_{i}\right)<1$ for some $i \in\{1,2,3\}$, say for $i=1$, then

$$
\frac{d^{\prime}\left(f(x), f\left(x_{1}\right)\right)}{d^{\prime}\left(f(x), f\left(x_{2}\right)\right)}=\frac{d^{\prime}\left(f(x), f\left(x_{1}\right)\right) d^{\prime}\left(f\left(x_{2}\right), f\left(x_{3}\right)\right)}{d^{\prime}\left(f(x), f\left(x_{2}\right)\right) d^{\prime}\left(f\left(x_{1}\right), f\left(x_{3}\right)\right)}=\frac{d\left(x, x_{1}\right) d\left(x_{2}, x_{3}\right)}{d\left(x, x_{2}\right) d\left(x_{1}, x_{3}\right)}<1 .
$$

Hence $d^{\prime}\left(f(x), f\left(x_{1}\right)\right)<d^{\prime}\left(f(x), f\left(x_{2}\right)\right)$. Since $d^{\prime}\left(f\left(x_{1}\right), f\left(x_{2}\right)\right)=1$, the strong triangle inequality shows that $d^{\prime}\left(f(x), f\left(x_{2}\right)\right)=1$ and hence $d^{\prime}\left(f(x), f\left(x_{1}\right)\right)=d\left(x, x_{1}\right)$.

Now let $x, y \in X$ be arbitrary points. Then there exists $i \in\{1,2,3\}$ such that $d\left(x, x_{i}\right)=d\left(y, x_{i}\right)=1$. It follows from what we have just proved that

$$
d^{\prime}\left(f(x), f\left(x_{i}\right)\right)=d\left(x, x_{i}\right)=1=d\left(y, x_{i}\right)=d^{\prime}\left(f(y), f\left(x_{i}\right)\right) .
$$

Hence for $j \neq i$ we have

$$
\frac{d^{\prime}(f(x), f(y))}{d^{\prime}\left(f(x), f\left(x_{j}\right)\right)}=\frac{d^{\prime}(f(x), f(y)) d^{\prime}\left(f\left(x_{j}\right), f\left(x_{i}\right)\right)}{d^{\prime}\left(f(x), f\left(x_{j}\right)\right) d^{\prime}\left(f(y), f\left(x_{i}\right)\right)}=\frac{d(x, y) d\left(x_{j}, x_{i}\right)}{d\left(x, x_{j}\right) d\left(y, x_{i}\right)}=\frac{d(x, y)}{d\left(x, x_{j}\right)}
$$

Since $d^{\prime}\left(f(x), f\left(x_{j}\right)\right)=d\left(x, x_{j}\right)$, we obtain $d^{\prime}(f(x), f(y))=d(x, y)$, as required.

Theorem 5.2. Let $(X, d)$ and $\left(X^{\prime}, d^{\prime}\right)$ be arbitrary ultrametric spaces and let $f: X \rightarrow X^{\prime}$ be a Möbius map. Then $f$ is a local similarity.

Proof. Choose a triple $p=\left\{p_{1}, p_{2}, p_{3}\right\}$ in $X$ and let $p^{\prime}=\left\{f\left(p_{1}\right), f\left(p_{2}\right), f\left(p_{3}\right)\right\}$. Consider the normalization $\left(X_{p}, \chi_{p}\right)$ of $(X, d)$ with respect to $p$. Similarly, consider the normalization $\left(X_{p^{\prime}}^{\prime}, \chi_{p^{\prime}}\right)$ of $\left(X^{\prime}, d^{\prime}\right)$ with respect to $p^{\prime}$. Since $\mathrm{I}_{p}:(X, d) \rightarrow\left(X_{p}, \chi_{p}\right)$ and $\mathrm{I}_{p^{\prime}}:\left(X^{\prime}, d^{\prime}\right) \rightarrow\left(X_{p^{\prime}}^{\prime}, \chi_{p^{\prime}}\right)$ are both Möbius maps and local similarities, the map $f: X \rightarrow X^{\prime}$ is a local similarity if and only if the map $f_{p, p^{\prime}}: X_{p} \rightarrow X_{p^{\prime}}^{\prime}$, defined by $f_{p, p^{\prime}}=\mathrm{I}_{p^{\prime}} \circ f \circ \mathrm{I}_{p}^{-1}$, is a local similarity. Since $f_{p, p^{\prime}}$ maps the diametrical triple $\left\{p_{1}, p_{2}, p_{3}\right\}$ to a diametrical triple $\left\{f\left(p_{1}\right), f\left(p_{2}\right), f\left(p_{3}\right)\right\}$, Lemma 5.1 implies that $f_{p, p^{\prime}}$ is an isometry. We conclude that the map $f=\mathrm{I}_{p^{\prime}}^{-1} \circ f_{p, p^{\prime}} \circ \mathrm{I}_{p}$ is a local similarity.

The proof of Theorem 5.2 yields the following two corollaries.

Corollary 5.3. Let $(X, d)$ and $\left(X^{\prime}, d^{\prime}\right)$ be arbitrary ultrametric spaces and let $p=\left\{p_{1}, p_{2}, p_{3}\right\}$ be any triple in $X$. If $f: X \rightarrow X^{\prime}$ is a Möbius map, then $f=$ $\mathrm{I}_{p^{\prime}}^{-1} \circ I_{p, p^{\prime}} \circ \mathrm{I}_{p}$, where $p^{\prime}=\left\{f\left(p_{1}\right), f\left(p_{2}\right), f\left(p_{3}\right)\right\}$ and where $I_{p, p^{\prime}}: X_{p} \rightarrow X_{p^{\prime}}^{\prime}$ is an isometry mapping the triple $p$ to the triple $p^{\prime}$.

Corollary 5.4. Let $(X, d)$ and $\left(X^{\prime}, d^{\prime}\right)$ be arbitrary ultrametric spaces and let $p=\left\{p_{1}, p_{2}, p_{3}\right\}$ be any triple in $X$. If $f: X \rightarrow X^{\prime}$ is a local similarity, then $f=$ $\mathrm{I}_{p^{\prime}}^{-1} \circ S_{p, p^{\prime}} \circ \mathrm{I}_{p}$, where $p^{\prime}=\left\{f\left(p_{1}\right), f\left(p_{2}\right), f\left(p_{3}\right)\right\}$ and where $S_{p, p^{\prime}}: X_{p} \rightarrow X_{p^{\prime}}^{\prime}$ is a local similarity mapping the triple $p$ to the triple $p^{\prime}$.

We end the paper with the following example. Let $(X, d)$ be a separable ultrametric space. As was proved by Timan and Vestfrid [17], every separable ultrametric space can be isometrically embedded in the space $l_{2}$ of all real sequences $\left\{x_{n}\right\}_{n \in \mathbf{N}}$, for which $\sum_{n \in \mathbf{N}} x_{n}^{2}<\infty$, with the norm $\|x\|=\left(\sum_{n \in \mathbf{N}} x_{n}^{2}\right)^{1 / 2}$. Let $f: X \rightarrow l_{2}$ be an isometric embedding such that $0 \notin f(X)$. The reflection

$$
I(x)=\frac{x}{\|x\|^{2}}
$$

is a Möbius map, that is,

$$
\frac{\|a-b|\||| c-d\|}{\|a-c|||| b-d\|}=\frac{\|I(a)-I(b)\|\|I(c)-I(d)\|}{\|I(a)-I(c)|\||| I(b)-I(d)\|}
$$


for $\{a, b, c, d\} \subset l_{2}$. This is known for the finite-dimensional Euclidean spaces (see, for example, [1]) and follows for $l_{2}$ by the consideration of four-dimensional $l^{\prime} \subset l_{2}$ meeting the condition $\{a, b, c, d\} \subset l^{\prime}$. Consequently, the map $I \circ f$ is an example of a Möbius map on $(X, d)$. It is interesting to note that for every $x \in l_{2}$ and $\epsilon>0$ the restriction of $I$ on the ball $B(x, \epsilon)$ is not a similarity.

Acknowledgement. The author would like to thank the referee for many valuable and constructive suggestions to improve the presentation as well as for suggesting to include the above example.

\section{References}

[1] Ahlfors, L. V.: Möbius transformations in several dimensions. - Ordway Professorship Lectures in Mathematics, Univ. of Minnesota, Minneapolis, 1981.

[2] Bestrina, M.: R-trees in topology, geometry, and group theory. - In: Handbook of geometric topology, edited by R. J. Daverman and R. B. Sher, North-Holland, Amsterdam, 2002, 55-91.

[3] Bonk, M., and O. Schramm: Embeddings of Gromov hyperbolic spaces. - Geom. Funct. Anal. 10, 2000, 266-306.

[4] David, G., and S. Semmes: Fractured fractals and broken dreams. - Oxford Lecture Ser. Math. Appl. 7, The Clarendon Press, Oxford Univ. Press, New York, 1997.

[5] Dragovich, B., A. Yu. Khrennikov, S. V. Kozyrev, and I. V. Volovich: On p-adic mathematical physics. - P-Adic Numbers Ultrametric Anal. Appl. 1:1, 2009, 1-17.

[6] Dordovskyi, D., O. Dovgoshey, and E. Petrov: Diameter and diametrical pairs of points in ultrametric spaces. - P-Adic Numbers Ultrametric Anal. Appl. 3:4, 2011, 253-262.

[7] Dovgoshey, O., and O. Martio: Blow up of balls and coverings in metric spaces. Manuscripta Math. 127, 2008, 89-120.

[8] Hughes, B.: Trees and ultrametric spaces: a categorical equivalence. - Adv. Math. 189:1, 2004, 148-191.

[9] Ibragimov, Z.: Hyperbolizing hyperspaces. - Michigan Math. J. 60, 2011, 215-239.

[10] KatoK, S.: p-adic analysis in comparison with real. - MASS Selecta: Teaching and Learning Advanced Undergraduate Mathematics 11-87, AMS, Providence, 2003.

[11] Koblitz, N.: p-adic numbers, p-adic analysis, and zeta-functions. - Springer, New York, 2nd edition, 1984.

[12] LApidus, M., and M. VAn FRAnKEnhuiJSEn: Fractal geometry, complex dimensions and zeta functions: Geometry and spectra of fractal strings. - Springer, New York, 2006.

[13] Lapidus, M., and H. Lu: Nonarchimedean Cantor set and string. - J. Fixed Point Theory Appl. 3, 2008, 181-190.

[14] Luosto, K.: Ultrametric spaces bi-Lipschitz embeddable in $\mathbf{R}^{n}$. - Fund. Math. 150:1, 1996, 25-42.

[15] Robert, A.: A course in p-adic analysis. - Springer, New York, 2000.

[16] Schikhof, W. H.: Ultrametric calculus : an introduction to p-adic analysis. - Cambridge Univ. Press, Cambridge, 1984.

[17] Timan, A.F., and I. A. VestFrid: Any separable ultrametric space can be isometrically embedded in $l_{2}$. - Funct. Anal. Appl. 17:1, 1983, 70-71; Translated from Funktsional. Anal. i Prilozhen. 17:1, 1983, 85-86.

[18] VÄIsÄLÄ, J.: Gromov hyperbolic spaces. - Expo. Math. 23:3, 2005, 187-231. 